\title{
BONDAD, COMPASIÓN Y VIRTUD: CLAVES DE LAS PROPUESTAS EDUCATIVAS DE ROUSSEAU ${ }^{\S}$
}

\author{
Goodness, Compassion and Virtue: Keys to Rousseau's \\ Education Proposals
}

\author{
Alicia Villar Ezcurra \\ Fecha de recepción: 18/03/2015 • Fecha de aceptación: 10/05/2015
}

Resumen. La defensa de la bondad originaria del ser humano es una de las concepciones más conocidas y polémicas del pensamiento de Rousseau. En este artículo se analiza la vinculación de su concepción antropológica con su modelo educativo. Al defender que el ser humano tiene una «disposición» al bien, es compasivo y se siente concernido ante el sufrimiento ajeno, Rousseau no piensa en un regreso al estado salvaje, sino en una educación que desarrolle dichas tendencias, mediante «las máximas de la piedad» y el ejercicio de la virtud. De este modo, propone una educación orientada al fomento de la cooperación y de los sentimientos humanitarios, que vea en el otro a un igual más que a un rival.

Finalmente, se reflexiona sobre la ambivalencia de la compasión y sobre algunas de las valoraciones de este sentimiento, considerado por Rousseau como origen de los sentimientos de humanidad.

Palabras clave: Rousseau. Bondad. Compasión. Virtud. Educación.

Abstract. The defense of the innate goodness of human beings is one of the most wellknown and polemic aspects of J. J. Rousseau's philosophy. This article analyses the link between his anthropological conception and his educational model. In defending the idea that the human being has a natural «disposition» for doing good, feeling compassion and empathizing with others' suffering, Rousseau is not invoking some long-lost, savage state of ours; rather, he is referring to a model of education that can help to encourage and develop such qualities by means of "maxims of pity» and the practice of virtue. In doing so he is proposing

\footnotetext{
$\S$ Este artículo se vincula con el Proyecto de Investigación del Plan Nacional de I+D del MINECO: «Fundamentos filosóficos de la idea de solidaridad: amor, amistad, generosidad» (FFFI 2012-37670).

* Universidad Pontificia de Comillas. Facultad de Ciencias Humanas y Sociales. Departamento de Filosofía, Humanidades y Comunicación. C/ Universidad Comillas, 3. 28049 Madrid. España. avillar@comillas.edu
}

Cómo citar este artículo: Villar Ezcurra, Alicia. «Bondad, compasión y virtud: Claves de las propuestas educativas de Rousseau», Historia y Memoria de la Educación, 2 (2015): 45-72. 
an education that encourages cooperation as well as humane feelings that will lead individuals to see others as equals rather than as rivals.

We also offer a reflection here on the ambivalence of compassion and on some of the assessments and opinions regarding this sentiment, which was considered by Rousseau to lie at the very origin of humane feelings.

Keywords: Rousseau. Goodness. Compassion. Virtue. Education.

\section{INTRODUCCIÓN}

A pesar de que Rousseau defendió que su pensamiento se derivaba de algunos pocos principios, sus contemporáneos pronto detectaron contradicciones en sus escritos. El mismo Voltaire observó el arte que aplicaba precisamente en escribir contra las artes, y receló de sus proclamas a favor del pueblo. Interpretó su elogio del estado de naturaleza como un regreso al estado salvaje, y vio su defensa de la bondad natural como la apología de una inocencia equivalente a la más completa estupidez.

A ello se sumaba el contraste entre los dichos y los hechos de la vida de Rousseau, lo que favoreció que se le tratara como un personaje extraño, desde un punto de vista intelectual, religioso y social. En realidad, Rousseau había comenzado por denunciar la violencia de los hombres poderosos y la opresión de los débiles en la sociedad de su tiempo, y apelaba a una experiencia continua como dispensa de prueba. ${ }^{1}$ En pleno Siglo de las Luces había observado que el buen sentido dependía más de los sentimientos del corazón que de las luces del espíritu; y a lo largo de la vida, había comprobado «que los más sabios y los más ilustrados no siempre son los más buenos, ni los que mejor se conducen en los asuntos de la vida» (Memoria presentada a M. de Mably, 1740). ${ }^{2}$

La bibliografía sobre su pensamiento es extensísima, de modo que sólo quisiera destacar aquí la vinculación de su tesis de la bondad natural con varios sentimientos que considera originarios: el sentimiento de piedad o compasión y el de justicia. Sobre estos presupuestos elabora su propuesta

\footnotetext{
${ }^{1}$ Jean-Jacques Rousseau, Discurso sobre el origen y los fundamentos de la desigualdad entre los hombres, en Del contrato social. Sobre las ciencias y las artes. Sobre el origen y los fundamentos de la desigualdad entre los hombres (Madrid: Alianza Editorial, 1989), 206 y 309. Traducción de Mauro Armiño. Las citas a estas tres obras se harán conforme a esta edición.

2 Jean-Jacques Rousseau, Ouvres Complètes II (Paris: Éditions du Seuil, L'Intégrale, 1971), 24.
} 
en el Emilio o sobre la Educación, con el propósito de que estas disposiciones naturales ni se perviertan, ni se acallen.

\section{LA ANTÍTESIS: HOMBRE NATURAL Y HOMBRE CIVIL}

Al querer mostrar que todos los vicios que se imputan al corazón humano no son naturales, el estudio de Rousseau sobre el ser humano está dominado por la oposición hombre natural-hombre civil. Dicha dicotomía ha de ser matizada, pues cada uno de estos tipos se divide a su vez en dos, lo que no siempre ha sido suficientemente subrayado.

Rousseau califica a los seres humanos como buenos ${ }^{3}$ en dos casos: uno es el del primitivo salvaje, libre, inocente y feliz, "esencialmente bueno" según el Discurso sobre el origen y los fundamentos de la desigualdad entre los hombres, en adelante citado como Segundo Discurso; el otro caso, se refiere al mundo civilizado, y se ejemplifica en Emilio, una persona auténtica y en este sentido natural, ${ }^{4}$ que crece al margen de la sociedad corrupta. Ambas figuras, el salvaje y Emilio, representan dos ejemplos de hombre natural, pues en la obra de Rousseau la palabra «natural» oscila permanentemente entre el sentido estático de simplicidad original y el sentido dinámico de perfección y autenticidad.

Además, la oposición entre el hombre salvaje y el hombre civilizado que aparece en el Segundo Discurso debe contrastarse con el resto de sus escritos políticos. En ellos defiende que la naturaleza humana sólo se desarrolla plenamente con una vida social debidamente orientada. ${ }^{5}$ Por tanto, sus observaciones sobre el hombre civil se desdoblan a su vez en dos: por un lado se refiere al que vive en la mera apariencia, el contra modelo descrito en los dos primeros Discursos y, por otro lado, al ciudadano que evoca en sus escritos políticos.

En el Discurso sobre las Ciencias y las Artes, su primer Discurso, Rousseau denunció que nuestras almas se habían corrompido a medida que las ciencias y las artes habían avanzado. Ciencias y artes, lejos de ser neutrales,

\footnotetext{
3 Cfr. Arthur M. Melzer, Rousseau. La bonté naturelle de l'homme (Paris: Belin, 1998), 36 y ss.

${ }^{4}$ Aquí Rousseau entiende por natural lo que es conforme a la verdadera naturaleza del hombre, lo que es esencial por oposición a lo accidental.

5 Cfr. Robert Derathé, «L’homme selon Rousseau», en P. Benichou, E. Cassirer, R. Derathé y otros autores, Pensée de Rousseau (Paris: Editions du Seuil, 1984), 109-125.
} 
distraen, hacen olvidar la pérdida de la libertad originaria e inalienable, de ahí su frase: "ciencias y artes extienden guirnaldas de flores sobre las cadenas de hierro que esclavizan a los hombres». ${ }^{6}$ A su juicio, la búsqueda del éxito y del reconocimiento arrastran un cúmulo de vicios: mentiras, rivalidades, envidias, odios y traiciones. De este modo, considera que el avance de las ciencias y las artes favoreció la corrupción de las antiguas costumbres y la pérdida de la virtud, secuelas del lujo característico de algunas sociedades civilizadas. Sin embargo, aunque Rousseau defiende y evoca repetidamente la palabra «virtud» a lo largo de su primer Discurso, no precisa su significación, que será distinta a lo largo de su obra. ${ }^{7}$ Es más claro cuando se refiere al vicio, que entiende como falsedad, engaño y apariencia de bien. ${ }^{8}$ Precisamente, en su primer Discurso la crítica a las sociedades civilizadas de su tiempo se centra en su falta de transparencia, ${ }^{9}$ su mentira, su encubrimiento de los vicios y de la corrupción.

Los contraejemplos a ese culto al artificio son los antiguos, aquellos que conciliaron la virtud y la felicidad, entre los que destaca la antigua Roma, los primeros persas, los germanos y los espartanos que se conservaron libres, independientes y moderados. En este Primer Discurso, virtud equivale a la renuncia a los vicios: la vanidad, la ociosidad y el lujo, y lamenta que los políticos de su tiempo no hablen más que de comercio y dinero, mientras que los antiguos hablaban sin cesar de costumbres y de virtud.

En la segunda parte de este primer Discurso, introducirá otro modelo frente a la corrupción de las sociedades de entonces: la vida en el estado natural, donde la transparencia era posible, por la simplicidad e inocencia:

[...] los hombres inocentes y virtuosos gustaban de tener a los dioses por testigos de sus acciones, moraban bajo las mismas cabañas; pero vueltos muy pronto malvados, se cansaron de aquellos incómodos espectadores y los relegaron a templos magníficos. ${ }^{10}$

\footnotetext{
${ }^{6}$ J. J. Rousseau, Discurso sobre las Ciencias y las Artes, 149.

7 J. J. Rousseau, Discurso sobre las Ciencias y las Artes, 165.

${ }^{8}$ Un estudio clásico sobre las distintas significaciones del término «virtud» en Rousseau es el de Albert Schinz, La pensée de Rousseau (París: Alcan, 1929).

${ }^{9}$ La búsqueda de la transparencia como una constante en el pensamiento de Rousseau ha sido analizada por Jean Starobinski. Jean-Jacques Rousseau. La transparencia y el obstáculo (Madrid: Taurus, 1983).
}

10 J. J. Rousseau, Discurso sobre las Ciencias y las Artes, 192. 
Años después desarrollará esta primera intuición en el Discurso sobre el origen y los fundamentos de la desigualdad entre los hombres; y presentará, a modo de hipótesis, el tránsito del estado de naturaleza al estado de sociedad como un proceso de desnaturalización y degeneración. Su propósito era denunciar la desigualdad como la raíz de profundas injusticias, que lleva a que la mayoría de los hombres sean instrumentos en manos de los que concentran mayor poder.

Mientras que en los dos primeros Discursos, Rousseau compara al hombre salvaje con lo que Robert Dérathé califica como «burgués inconsistente», en otros escritos ganará peso el modelo del antiguo ciudadano. Será constante en denunciar las pésimas instituciones de los pueblos modernos, y evocará las «sublimes instituciones» de la Antigüedad, Esparta y la antigua Roma, donde destacaba el valor y las virtudes cívicas. En Del Contrato Social describirá los principios políticos del nuevo modelo: la organización de un Estado legítimo, regido por unas leyes que expresan la voluntad general, donde los ciudadanos se orientan al ejercicio de las virtudes y los sentimientos de ciudadanía. Por este motivo, son capaces de sacrificar sus intereses particulares por el bien del todo del que forman parte, el bien común. Así, el lector de Rousseau comprueba que la palabra bondad, tan central en su caracterización del ser humano, apenas aparecerá en sus escritos políticos, donde evoca la virtud frente al vicio y la corrupción, y entiende por virtud la fuerza y el vigor del alma.

\section{AMOR DE SÍ Y PIEDAD EN LA VIDA NATURAL ${ }^{11}$}

En el Discurso sobre el origen y los fundamentos de la desigualdad entre los hombres, Rousseau mostró indirectamente la bondad natural al postular el origen exterior, social, de todos los vicios (segunda parte del Discurso); la prueba positiva consistirá en mostrar con distintos argumentos que el principio fundamental de nuestra naturaleza es bueno (primera parte del Discurso).

Rousseau se confronta con la visión de Hobbes sobre la vida en la naturaleza calificada como solitaria, pobre, tosca, embrutecida y breve. En un primer momento, para Rousseau la vida natural también es solitaria, simple

\footnotetext{
${ }^{11}$ Algunas de las cuestiones aquí abordadas se presentaron en el Congreso Internacional sobre Rousseau, celebrado en Murcia (octubre de 2012), publicadas en el capítulo «Compasión y amor a la justicia en Rousseau», en El legado de Rousseau. 1712-2012, eds. José López Hernández y Antonio Campillo Meseguer (Murcia, EDITUM, 2013), 235-251.
} 
y embrutecida, pues el ser humano parece limitado a las puras sensaciones, y sólo se distingue de ellos por su libertad y su facultad de perfeccionarse, lo que llama perfectibilidad, entendida como maleabilidad, plasticidad. ${ }^{12}$ Con ello reemplaza la definición clásica del hombre como animal racional por la definición del hombre como agente libre. ${ }^{13}$ En el estado de naturaleza la razón, la imaginación y la conciencia, aún no se han desarrollado en el ser humano; sin embargo ello no implica que su vida sea necesariamente violenta y cruel. Como Hobbes, Rousseau reconoce la importancia de las pasiones como motor de las acciones humanas, pero le reprocha haber atribuido al estado de naturaleza los vicios que se desarrollan en el estado civil. ${ }^{14}$ Así, el modelo del hombre natural del segundo Discurso no alude simplemente a la vida primitiva de los salvajes, sino que responde a la idea de la humanidad incorrupta, contrapuesta a la opresión que ha prevalecido en el desarrollo de la sociedad civil.

En el estado de naturaleza el ser humano es bueno con relación a sí mismo, en tanto sus deseos son proporcionales a sus necesidades; entonces es autosuficiente, fuerte, independiente y feliz. El hombre natural vive el presente, disfruta de sí mismo y de su existencia, sin importarle lo que piensen los demás. Goza de independencia, no es esclavo de la opinión ajena, ni se crea necesidades superfluas. Es un ser habituado a soportar adversidades y dificultades, fuerte, con escasa relaciones con otros hombres, de ahí que no pueda decirse que cuente propiamente con una moralidad, pues la calma de las pasiones y la ignorancia de los vicios le impiden obrar mal. En el estado de naturaleza, el ser humano, como Adán, no conoce el árbol del bien y del mal, y por tanto no tiene ni virtudes, ni vicios, ${ }^{15}$ como el niño, su inocencia es ignorancia.

Con respecto a su relación con los demás, la afirmación de Rousseau de que el hombre es bueno tiene varios sentidos. Un primer sentido consiste

\footnotetext{
12 Rousseau, Discurso sobre el origen y los fundamentos de la desigualdad entre los hombres, 220. La palabra «perfectibilidad» (perfectibilité) es un neologismo creado por Rousseau para resaltar que las circunstancias conforman al ser humano.

${ }^{13}$ Cfr. Leo Strauss, «L'intention de Rousseau», en P. Benichou, E. Cassirer, R. Derathé y otros autores, Pensée de Rousseau (Paris: Editions du Seuil, 1984), 94.

${ }_{14}$ Para Hobbes la guerra de todos contra todos es una consecuencia de la igualdad natural. Th. Hobbes, Leviatán (Madrid: Tecnos, 1989), XIII, 123-125. Traducción de E. Tierno Galván y M. Sánchez Sarto. Las citas a esta obra se harán por esta edición.

15 J. J. Rousseau, Discurso sobre el origen y los fundamentos de la desigualdad entre los hombres, 233.
} 
en no querer perjudicar a otro, pues en esa situación el ser humano carece de las relaciones y del cúmulo de necesidades que conducen al conflicto. Pero además para Rousseau la bondad tiene otro sentido que se vincula con el sentimiento de piedad o compasión, que nos lleva a identificarnos con la persona a la que vemos sufrir. En el segundo Discurso Rousseau empleó preferentemente el término pieté y ocasionalmente los términos conmiseration y compassion, para defender su tesis de que «el hombre es naturalmente bueno», ${ }^{16}$ pues al experimentar sentimientos de compasión, neutraliza su violencia y se aparta de la crueldad.

Como es sabido, Rousseau distingue dos principios en el ser humano: «el amor de sí», o "el amor de sí mismo», y el "amor propio» (amour prope), ${ }^{17}$ expresión que en el francés clásico significa egoísmo. En Bernard de Mandeville también aparecía la distinción entre self love y self liking como dos pasiones naturales; pero si el primero estaba ligado a la necesidad, el segundo se vinculaba al deseo vanidoso. ${ }^{18}$ Rousseau variará el análisis de Mandeville, y describe el amor de sí como el principio fundamental de la naturaleza humana, instinto propio de todo ser viviente, y que permite que el ser humano disfrute de su existencia, la conserve y mejore. Considera que es connatural al hombre a diferencia del amor propio, pasión desnaturalizada que modifica el amor de sí y que lleva a que cada individuo se crea el centro de todo, de ahí que inspire a los hombres todos los males que mutuamente se hacen. Mientras que el amor de sí se contenta cuando nuestras verdaderas necesidades son satisfechas, el amor propio se compara con los demás y nunca está satisfecho. Implica preferirse a los demás y exige que los demás no se prefieran así mismos, lo que resulta del todo punto imposible. El amor propio convierte al ser humano en envidioso y codicioso, y es el origen de todos los vicios que Hobbes atribuía al hombre en el estado de naturaleza, y el motor del incesante afán de poder que propicia una atmósfera de enfrentamiento y conflicto, que llamará estado de guerra.

A diferencia de Hobbes, para Rousseau el amor de sí es connatural al hombre, mientras que el amor propio no es original sino que se adquiere

\footnotetext{
${ }^{16}$ La expresión «bondad original», por oposición a «perversidad original», se encuentra en la carta a M. de Beaumont, mientras que la expresión «el hombre es naturalmente bueno» se incluye en el segundo Discurso y en la carta de Rousseau a Philopolis.

17 La distinción aparece en la nota quince del Discurso sobre el origen y los fundamentos de la desigualdad entre los hombres, 329-330.

18 Bernard de Mandeville, La fábula de las abejas (México: FCE, 1982), 2. ${ }^{\text {a }}$ parte, tercer diálogo, 439.
} 
en una vida en sociedad, donde prevalece la ambición y la rivalidad. En el Emilio, Rousseau señalará que las pasiones afectuosas nacen del amor de sí, mientras que las irascibles nacen del amor propio. Como pasión, el amor de sí es siempre bueno y conforme al orden, pues para conservarnos es preciso amarnos y, al tiempo, amar lo que nos conserva. ${ }^{19}$ Con relación al amor de sí, Rousseau aborda la conmiseración o piedad por primera vez en el Prefacio del segundo Discurso. Nos dice:

Creo percibir dos principios anteriores a la razón, uno de los cuales nos hace interesarnos ardientemente por nuestro bienestar y por la conservación de nosotros mismos, y el otro nos inspira una repugnancia natural a ver perecer o sufrir a cualquier ser sensible y principalmente a nuestros semejantes. Del concurso y de la combinación que nuestro espíritu es capaz de hacer de estos dos principios, sin que sea necesario hacer entrar ahí el de la sociabilidad, es de donde me parece que derivan todas las reglas del derecho natural $[\ldots]$

Y prosigue líneas después:

[...] mientras no oponga resistencia al impulso interior de la conmiseración, [el ser humano] jamás hará daño a otro hombre, ni siquiera a ningún ser sensible, salvo en el caso legítimo en el que, hallándose interesada su conservación, está obligado a darse preferencia a sí mismo $\left[\ldots . .{ }^{20}\right.$

Al plantear la cuestión de la ley natural, observa que para que sea natural es preciso que "hable de modo inmediato por voz de la naturaleza». ${ }^{21}$ Por tanto, si estoy obligado a no hacer ningún mal a nuestro semejante es más por ser sensible que por ser razonable. A diferencia de los racionalistas, para Rousseau existir es sentir (Emilio o sobre la Educación) y la sensibilidad es el principio de toda acción que expresa la voz de la naturaleza (Rousseau, juez de Jean-Jacques). El amor de sí y la piedad son dos sentimientos que se apoyan en nuestra condición de seres sensibles, son puros

\footnotetext{
${ }^{19}$ En el libro IV del Emilio o de la Educación considera que el amor de sí es el origen de todas las pasiones, pues es anterior a todas las demás, que son, de algún modo, modificaciones posteriores (Madrid: Alianza Editorial, 1998), 285. Las citas a esta obra se harán conforme a esta edición.

20 J. J. Rousseau, Discurso sobre el origen y los fundamentos de la desigualdad entre los hombres, 198-199.

21 J. J. Rousseau, Discurso sobre el origen y los fundamentos de la desigualdad entre los hombres, 198.
} 
movimientos de la naturaleza, anteriores al desarrollo de la reflexión y la racionalidad.

El amor de sí que nos hace interesarnos «sobremanera» en nuestro propio bienestar establece una rudimentaria interioridad, mientras que la piedad permite descubrir la alteridad. ${ }^{22}$ En esta primera referencia a la compasión, del Prefacio del segundo Discurso, no alude al sufrimiento inmerecido de aquel al que compadecemos. Sin entrar en la causa del sufrimiento, su percepción nos produce inmediatamente pesar al descubrir al otro, y a mí mismo en él, en lo que tenemos de común: la fragilidad. «Son nuestras miserias comunes, lo que lleva nuestros corazones a la humanidad [...] si cada uno de nosotros no tuviéramos ninguna necesidad de los demás, apenas pensaríamos en unirnos a ellos», dirá en el Emilio. ${ }^{23}$

Más adelante, en la primera parte de este mismo Discurso vuelve a abordar la piedad, en esta ocasión como una refutación expresa de Hobbes. El autor del Leviatán había definido la compasión como «el dolor que causa una desgracia o calamidad ajena», y señalaba que se producía por «la idea de que una desgracia similar nos pueda ocurrir a nosotros mismos». ${ }^{24}$ Por tanto, la compasión se asociaba directamente con el temor e indirectamente con el egoísmo, de ahí que ni la compasión ni la bondad ejerzan función efectiva, ni correctiva, en su posterior descripción del estado de naturaleza como un estado de guerra. En cambio Rousseau, en un largo pasaje que Schopenhauer trascribirá en su obra Sobre el fundamento de la moral como su antecedente filosófico más directo, caracteriza a la piedad como la única "virtud natural» y resalta la importancia y utilidad de sus efectos. ${ }^{25}$ Sin explicitar su teísmo, observa que la piedad, que ha sido dada al ser humano para suavizar, templar o atemperar el ardor que tiene por su propio bienestar, hace sentir una repugnancia innata a ver sufrir al semejante.

En resumen, a lo largo del segundo Discurso Rousseau considera a la piedad como un principio del alma, después como un impulso, más adelan-

\footnotetext{
22 Cfr. Raymond Trousson et Frédéric S. Eigeldinger (dirs), Dictionnaire de Jean-Jacques Rousseau (Paris: Honoré Champion, 2006), y J. Starobinski, Jean-Jacques Rousseau, 723 (voz: «Pitié»).

23 J. J. Rousseau, Oeuvres Complètes, II, 295.

24 Th. Hobbes, Leviatán, VI, 88.

25 A. Schopenhauer, «Sobre el fundamento de la moral», en Los dos problemas fundamentales de la ética (Madrid: Siglo XXI, 2002), 271. Traducción de Pilar López Santamaría.
} 
te como la «única» virtud natural y universal y como "puro movimiento de la naturaleza», y por último como un "sentimiento natural». ${ }^{26} \mathrm{Su}$ función de «movimiento», de "impulso», su dinamismo es central, pues explica que de hecho contrapese la fuerza del otro principio esencial: el amor de sí. La utilidad y función de la compasión es equilibrar y regular ese amor de sí y es tanto más útil cuanto precede al uso de la razón y a la reflexión. Es anterior a todo cálculo ${ }^{27}$ y es una «disposición» conveniente a seres tan débiles y tan sujetos a males como somos, pues favorece la supervivencia de la especie. La piedad dicta la máxima de bondad natural: «haz tu bien con el menor mal posible para el otro», y en el estado de naturaleza «ocupa el lugar de las leyes, las costumbres y la virtud». ${ }^{28}$ Consiste en sufrir «con», condolerse del sufrimiento o miseria ajena como recoge el término "conmiseración», y requiere "ver» el sufrimiento, según el segundo Discurso, ya que ni la imaginación ni la razón aún se ha desarrollado. En cambio, en el Discurso sobre el origen de las lenguas y en el Emilio Rousseau destacará la importancia de la imaginación para representarse la desgracia ajena, pues "es en otro en quien sufrimos», lo que muestra la evolución de este tema a lo largo de su obra.

Volviendo al texto del Segundo Discurso, para apoyar y confirmar su tesis del carácter natural de la compasión, invoca el reconocimiento del que califica como «el mayor detractor de las virtudes humanas», Mandeville, y resume un ejemplo de piedad ofrecido por este autor. ${ }^{29} \mathrm{~A}$ la cita de Mandeville sigue una confirmación por parte de Rousseau, por medio de una nueva prueba. Consiste en recurrir a ejemplos de la vida animal, cosa que ya había señalado Montaigne leyendo a los clásicos y que hoy comprueban científicos como Antonio Damasio. ${ }^{30}$ Los dos ejemplos de la vida animal que Rousseau cita son: «la ternura de las madres por sus pequeños» $\mathrm{y}$ «la repug-

\footnotetext{
${ }^{26}$ Después de la crítica de Locke a la existencia de las ideas innatas, el término «innato» parece evitarse por parte de los ilustrados que admiraban en gran parte su obra.

${ }^{27}$ Para Hobbes la razón también es la capacidad propia del individuo para hacer cálculos. Entiende el razonamiento como cálculo y la actividad racional, como el cálculo de los medios adecuados para alcanzar los objetivos deseados (Leviatán, cap. V: «De la razón y de la ciencia», 73).

${ }^{28}$ Rousseau, Discurso sobre el origen y los fundamentos de la desigualdad entre los hombres, 239.

${ }^{29}$ Adam Smith observó que gracias a Rousseau las principales ideas del libertino Mandeville «aparecieron con toda la pureza y sublimidad de Platón», Edinburgh Review (1755); cfr. Melzer, Rousseau. La bonté naturelle de l'homme, 53, nota 15.

${ }^{30}$ Antonio Damasio, En busca de Spinoza. Neurobiología de la emoción y los sentimientos (Barcelona: Drakontos, 2009), 156
} 
nancia que tienen los caballos por pisar un cuerpo vivo». ${ }^{31}$ Aquí no advierte que la compasión presupone que el hombre es un ser empático, que dispone de lo que Montaigne y los moralistas británicos llamaron simpatía. ${ }^{32}$ En realidad, como precisarán los teóricos y fenomenólogos de los sentimientos morales, desde a Adam Smith hasta Max Scheler, la compasión es una de las formas de la simpatía, es la empatía con el dolor o la tristeza del otro. En cambio, según el segundo Discurso, el ser humano descubre la alteridad precisamente cuando experimenta compasión.

Rousseau quiere así destacar lo que a su juicio Mandeville, ni ningún otro, observó: que de la sola compasión se desprenden todas las virtudes sociales, y defiende su originalidad en este punto, al señalar que de ahí se derivan todas las reglas de derecho natural. ${ }^{33}$ Las virtudes de generosidad, de clemencia y de humanidad, entendida como una piedad aplicada a los débiles, a los culpables o a la especie humana en general, la benevolencia e incluso la amistad, son producto de una piedad constante fijada sobre un objeto en particular, pues desear que alguien no sufra más es querer que alguien sea feliz, lo que se ha llamado la cara alegre de la compasión. ${ }^{34}$ Con ello, se cumple el propósito inicial que Rousseau había señalado en el Prefacio del segundo Discurso: deducir a partir de los primeros principios todas las reglas de derecho natural.

En resumen, según este texto la compasión puede ser considerada como el fundamento de la moral, según la expresión acuñada por Schopenhauer, quien calificó a Rousseau como el mayor moralista de todos los tiempos, y su precedente filosófico más directo. Para ambos compadecer es sentir-con, lo que suprime la distancia entre los seres humanos que establece el egoísmo. Implica des-centrarse, convertir al otro en el centro de atención, sentirse concernido por su sufrimiento. Por su dinamismo y fuerza, en el estado de naturaleza juega el papel de las leyes, de las costumbres y de la virtud.

\footnotetext{
31 J. J. Rousseau, Discurso sobre el origen y los fundamentos de la desigualdad entre los hombres, 236.

32 Montaigne considera que «hay ciertas inclinaciones afectivas que a veces nacen sin el consejo de la razón, que vienen de una fortuita ligereza que otros llaman simpatía; son los animales tan capaces de ellas como nosotros». Michel de Montaigne, Ensayos, II, 12 (Madrid: Cátedra, 2006), 173.

33 J. J. Rousseau, Discurso sobre el origen y los fundamentos de la desigualdad entre los hombres 238.

34 Cfr. Aurelio Arteta, La compasión: una virtud bajo sospecha (Barcelona: Paidós, 1996).
} 
En realidad, como Schopenhauer, autor al que volveremos al final, Rousseau distingue dos niveles en la compasión. En el primero se evita hacer el mal, o al menos se frena o limita al «menor mal» según la máxima citada del Discurso; en el segundo nivel se trata de ayudar a quienes lo necesitan, función y dinamismo que promoverá en el Emilio. Volviendo al segundo Discurso, para Rousseau la piedad, en lugar de la máxima sublime de justicia razonada: "haz a otro lo que quieras que te hagan a ti», inspira a los hombres otra máxima de bondad natural, menos perfecta pero puede que más útil: "haz tu bien con el menor mal posible al otro». ${ }^{35}$

Ahora bien, considerar la compasión como un principio natural y universal, y constatar al tiempo la maldad de las sociedades civilizadas, obliga a Rousseau a explicar por qué motivo se altera y qué podemos hacer para evitarlo. Su tesis es que la compasión se debilita en las sociedades corruptas, debido a la fuerza del egoísmo, que repliega al ser humano sobre sí mismo y le distancia de todo lo que le aflige y molesta. Entonces el ser humano ya no es capaz de oír la voz de la naturaleza, su razón engendra el amor propio, y la reflexión lo fortifica y justifica. Este es el sentido de la polémica frase anti intelectualista sobre la insensibilidad, que al parecer le sugirió Diderot:

Se puede degollar impunemente a un semejante bajo su ventana; no tiene más que taparse los oídos y argumentar un poco, para impedir a la naturaleza, que se revuelve en él, identificarle con ése a quien se asesina. ${ }^{36}$

Con ello subraya el peligro del ejercicio de una razón instrumental, que se distancia del otro hasta llegar a la insensibilidad. En una sociedad basada en la desigualdad y en los intereses de los más poderosos, se favorece la transformación del amor de sí en amor propio o egoísmo, e impera la ley del más fuerte. Como en Hobbes, la razón aparece como un mecanismo de cálculo que orienta sobre los medios adecuados para lograr determinados fines a cualquier precio; de ahí su frase: «es la razón la que engendra el amor propio; y es la reflexión la que lo fortifica; es ella la que repliega al hombre sobre sí mismo». ${ }^{37}$

\footnotetext{
35 J. J. Rousseau, Discurso sobre el origen y los fundamentos de la desigualdad entre los hombres, 240.

36 J. J. Rousseau, Discurso sobre el origen y los fundamentos de la desigualdad entre los hombres, 239.

37 J. J. Rousseau, Discurso sobre el origen y los fundamentos de la desigualdad entre los hombres, 238.
} 
En la segunda parte del Discurso, explicará las consecuencias de la expansión del egoísmo, observando que desde el instante en el que un hombre se dio cuenta, calculó, que era útil acaparar provisiones para dos, se introdujo la propiedad y la desigualdad, y entonces la servidumbre y la miseria germinaron y crecieron. El inicial equilibrio entre el amor de sí y la piedad se perdió, y se instauró la ley del más fuerte, un hecho que no constituye derecho. ${ }^{38}$ La toma de conciencia de esta situación inspirará a Rousseau a buscar los remedios adecuados, pues si lo específicamente humano es la libertad y la perfectibilidad, es preciso buscar las condiciones y los medios para su pleno desarrollo. Como Kant advirtió, la descripción del estado de naturaleza por parte de Rousseau tenía un carácter regulativo más que descriptivo, que le llevó a proponer un modelo de educación y de organización social y política legítimas, que llevara a plenitud la dimensión moral del ser humano.

La cuestión: cómo evitar que un principio natural se destruya o se convierta en ineficaz,,$^{39}$ será abordada en el Emilio pero antes había sido tratada en las Cartas que Rousseau dirigió a Sofía de d'Houdetot en 1757 y 1758, las llamadas Cartas morales o Cartas a Sofía. ${ }^{40}$ La quinta carta resulta de una importancia especial, pues muchos de sus párrafos serán transcritos con variantes en la Profesión de fe del vicario saboyano. ${ }^{41}$ Ahí Rousseau reconocerá una capacidad de identificación con el otro que en circunstancias adecuadas permite extender a otros seres humanos el amor de sí. Una vez desarrollada la conciencia, estos sentimientos perviven en lo que llama la «voz de la naturaleza», una voz interior que requiere una serie de condiciones para poder ser oída.

\footnotetext{
38 J. J. Rousseau, Del Contrato social (Madrid: Alianza Editorial, 1988), 30. Traducción de Mauro Armiño.

39 En la segunda parte del Discurso, al tiempo que señala las sucesivas formas de socialización, Rousseau marcará las etapas de la alteración de la piedad natural: «La bondad, conveniente al puro estado de naturaleza, ya no era la que convenía a la sociedad naciente, era preciso castigos más severos cuando las ofensas eran más frecuentes y el terror de las venganzas ocupaba el lugar del freno de las leyes» (Discurso sobre el origen y los fundamentos de la desigualdad entre los hombres, 257).

${ }^{40}$ En las cartas que Rousseau escribió a Madame D`Houdetot, a quien llama Sofía, desde noviembre de 1757 hasta febrero de 1758, se propuso resumir sus principios filosóficos y morales; de ahí que en una carta de 28 de enero de 1758 las mencione como "Cartas morales». Cfr. Jean-Jacques Rousseau. Cartas a Sofía. Correspondencia filosófica y sentimental (Madrid: Alianza Editorial, 1999), edición y Estudio preliminar (9-76) de Alicia Villar. Las citas a esta obra se harán conforme a esta edición.

${ }^{41}$ Para desarrollar algunas de las ideas de la Profesión de fe del vicario, recurrirá a la quinta carta a Sofía, pero tachará su nombre, que sustituirá por la paternal fórmula de «hijo mío». El vicario será descrito como «un hombre por naturaleza humanitario y compasivo, que siente las penas ajenas como propias, y al que el bienestar no había endurecido el corazón» (Emilio o de la Educación, 353).
} 


\section{EL SENTIMIENTO DE JUSTICIA}

En sus Cartas morales o Cartas a Sofía, al exponer los principios fundamentales de su filosofía práctica, Rousseau defendió la existencia de un sentimiento innato de justicia, además de la piedad natural. Su postura se enfrentaba con la moral descrita en Francia fundamentalmente por Helvetius en su obra Sobre el espíritu (1758). Helvetius mantenía que el interés personal es un motor neutro en sí mismo, capaz de efectos buenos o malos para la sociedad; por tanto, no es que los seres humanos sean malvados, sino que están sometidos a sus propios intereses. Por el contrario, Rousseau afirma que «toda la moralidad de la vida humana está en la intención del hombre», ${ }^{42}$ frase de Pufendorf que retomará Kant. La calificación moral de una acción no depende de su resultado, sino del motivo, ${ }^{43}$ de la intención o resorte interno por el que se lleva a cabo. En el segundo Discurso, la bondad natural se asociaba a la compasión; sin embargo, aquí el bien se vincula sobre todo con el amor a la justicia.

Rousseau también insiste, en esta carta, en que no quiere entrar en discusiones metafísicas que no llevan a nada y afirma que «existir es para nosotros sentir» ${ }^{44}$ modificando el enunciado cartesiano para orientar la existencia humana hacia la vida moral. Le interesa defender el carácter innato de ciertos sentimientos que la causa de nuestra existencia, sea cual sea, ha provisto para la conservación de la especie.

No acude a un código religioso o social determinados y defiende una bondad y virtud practicable por todos, con independencia de los imperativos teológicos entonces aún vigentes. El juicio moral pertenece a cada individuo y no se asigna ningún papel al pecado original, como en los demás philosophes. La naturaleza en general, como comienza el Emilio, y la naturaleza humana en particular, es fundamentalmente buena y lo que nos deprava es la corrupción generalizada de las sociedades civilizadas. Como observa Charles Taylor, la imagen agustiniana de la voluntad, de una voluntad depravada, el amor sui, se comprueba en una doctrina que niega uno de los

\footnotetext{
${ }^{42} \mathrm{Al}$ comienzo de la quinta carta, Rousseau dudó entre «intención» $\mathrm{y}$ «voluntad», palabra que finalmente tachó. J. J. Rousseau, Cartas a Sofía, 127.

${ }^{43}$ Para el ginebrino, como para después Schopenhauer, no hay acción moral si es expresión del egoísmo.

44 J. J. Rousseau, Cartas a Sofía, 125.
} 
dogmas centrales de la teología agustiniana. ${ }^{45}$ Así, Rousseau traspone el esquema inocencia-caída-corrupción a términos seculares que corresponden a la tríada vida natural-sociedades corruptas-nuevo orden social y político, y para ello la educación resulta clave.

En la quinta carta a Sofía, como después en el Emilio, Rousseau defiende fácticamente la bondad, de un modo introspectivo y con varias experiencias. ${ }^{46}$ La primera es la satisfacción interior al practicar la justicia, lo que muestra que el bien debe estar en el fondo de nuestros corazones. De nuevo refuta a Hobbes, ahora implícitamente pues observa que si el ser humano "fuera como el lobo», ${ }^{47}$ hecho para cazar a su presa y dañar a sus semejantes, la conducta virtuosa suscitaría remordimientos y la bondad sería un vicio contra natura. ${ }^{48}$ Por tanto, la existencia de los remordimientos confirma la bondad originaria.

Con el fin de mostrar un nuevo argumento a favor de la bondad, Rousseau evoca de nuevo la experiencia de la compasión, ${ }^{49}$ y apela a la imaginación y a la memoria de su interlocutor. Describe varias situaciones que nos llevan a identificarnos con las víctimas. De un lado, la experiencia de ser espectador de una obra de teatro, ${ }^{50}$ ejemplo que tendrá una significación distinta en la Carta a d'Alembert, y, por otra parte, la de ser espectador de un acto de violencia en la calle. En ambos casos, se trata de la reacción ante el espectáculo de una desgracia, en el arte o en la vida real. Como en el segun-

\footnotetext{
${ }^{45}$ Cfr. Ch. Taylor, Fuentes del yo. La construcción de la identidad en la modernidad (Barcelona: Paidos,1996), 488.

${ }^{46}$ Para Rousseau la introspección revela la verdad del hombre, conclusión a la que también había llegado Hobbes cuando en un pasaje de la Introducción del Leviatán dice a su lector que lea en sí mismo a la humanidad, pues ese género de doctrina no admite otra demostración (Leviatán, 48).

47 J. J. Rousseau, Cartas a Sofía, 121.

${ }^{48}$ La fórmula "el hombre es naturalmente bueno» se encontraba en el segundo Discurso (nota 9) y también en la carta escrita a Philopolis. En cambio, en la Carta al Arzobispo C. de Beaumont (1763) escribirá bondad original por oposición a la perversidad original. En el Emilio, Rousseau presenta la inocencia del niño que reside en la ignorancia de los vicios más que en la bondad.

${ }^{49}$ La palabra piedad no aparece expresamente en las Cartas morales, pues fue tachada del manuscrito original. J. J. Rousseau, Cartas a Sofía, 128.

${ }^{50}$ En el segundo Discurso, Rousseau también menciona esta experiencia, abandonando por tanto su análisis del estado natural al introducir un ejemplo del mundo civilizado que recuperará en el Emilio. El análisis de las reacciones del espectador de las Tragedias había sido ya desarrollado por Aristóteles (Poética y Retórica). Para un desarrollo de este tema véase Alicia Villar, «El valor de la compasión en la Modernidad», en Alicia Villar y Miguel García-Baró, Pensar la solidaridad (Madrid: Universidad Pontificia Comillas, 2004), 124-129.
} 
do Discurso, aquí Rousseau repite que en los espectáculos nos identificamos con el héroe desdichado más que con el tirano triunfante..$^{51}$

También se detiene en la experiencia de compasión ante la visión de un "acto de violencia o injusticia en una calle o en un camino», ${ }^{52}$ que adquiere el valor de prueba y de confirmación. Se comprueba un giro con respecto al análisis del segundo Discurso, pues ahora no se trata sin más de la compasión ante el sufrimiento ajeno, sino del sufrimiento inmerecido que es causado por otro, por un tercero que es moralmente responsable de una desgracia. En este caso, el dolor causado no es consecuencia de un acontecimiento fortuito, sino de una acción reprobable que exige reparación. Entonces, de un modo inmediato, brota un sentimiento de cólera y de indignación en quien es testigo de un acto de violencia o de injusticia, dos sentimientos que sin ser positivos, nos movilizan e impulsan a ponernos al lado del oprimido.

Ello revela la complejidad de la compasión y la pluralidad de sentimientos que suscita: tristeza por la víctima, cólera, indignación y reprobación ante el agresor. A pesar de la pluralidad de sentimientos, la compasión se experimenta de un modo inmediato: "al instante» precisa Rousseau, ${ }^{53}$ e insiste en su dinamismo y su función motora. Todo ello le muestra que mientras el ser humano sea sensible, se identifique con las víctimas y no con sus verdugos, mientras no se sienta indiferente o insensible ante la visión del sufrimiento que provoca una injusticia, aún no ha perdido su sensibilidad moral.

La última experiencia que Rousseau explora en la quinta «Carta moral» es la culpabilidad, el arrepentimiento, un hecho que nos permite descubrir nuestra dimensión moral. Padecemos los remordimientos que nos atormentan, y nos gustaría suprimirlos pues nos hacen lamentar acciones, realizadas en un pasado que no podemos cambiar. Por este motivo, Rousseau cree que el malvado evita la soledad y busca entretenerse para huir

\footnotetext{
${ }^{51}$ La significación de esta experiencia variará por completo en la Carta a D’Alembert sobre los espectáculos. Ahí considera que la piedad del espectador es una «emoción pasajera y vana» que no dura más que la ilusión que la produce. En este caso se trata de una "piedad estéril y artificiosa» que no produce el más mínimo acto de humanidad, posibilidad que hasta entonces no había contemplado.

52 J. J. Rousseau, Cartas a Sofía, 122.

53 J. J. Rousseau, Cartas a Sofía, 122.
} 
del pesar. ${ }^{54}$ En realidad las reflexiones de Rousseau se asemejan mucho a las del Poema sobre la ley Natural de Voltaire que había leído un año antes y a quien no cita. ${ }^{55}$ Como Voltaire, Rousseau alude a las objeciones de los filósofos que mantienen que la conciencia moral es fruto de los prejuicios inculcados desde la infancia. ${ }^{56}$ Pero ambos defienden las ideas de justicia y honestidad y los principios de moral se dan en todas las naciones del mundo. Las excepciones no resultan relevantes, pues no hay un lugar donde sea un crimen "guardar su fe, ser clemente, bienhechor y generoso", ${ }^{57} \mathrm{y}$ donde la persona de bien sea despreciada y el malhechor apreciado. Rechaza «las disputas metafísicas que no llevan a nada», pues su procedimiento consiste en «hablar al corazón ${ }^{58}$ de su interlocutor, a partir de experiencias compartidas.

La conclusión insiste en el mismo inicio de la quinta Carta: «Hay un principio innato de justicia y de verdad en el fondo de nuestros corazones [...], por el cual juzgamos nuestras acciones y las de los demás como buenas o malas. Doy el nombre de conciencia a este principio». ${ }^{59}$

La conciencia, nuestro guía interior, habla la voz de la naturaleza, pero para oírla y llegar al fondo de nuestros corazones se precisan una serie de condiciones que Rousseau detallará a Sofía en su sexta Carta: liberarse de los prejuicios y de la dependencia de la opinión ajena, el mal de la época; también conviene realizar pequeños retiros y una cierta soledad en contacto con la naturaleza. Por último, la práctica de la beneficencia que nos hará desterrar el afán por lo superfluo y la vanidad. En definitiva, sólo después de hacer el bien se alcanzará la felicidad que procura la buena conciencia. Como para Aristóteles, es preciso la práctica, el hábito

\footnotetext{
${ }^{54}$ J. J. Rousseau, Cartas a Sofía, Quinta Carta, 122: «El malvado huye de sí mismo y se arroja al exterior [...]; por el contrario la serenidad del justo es interior. Sólo está tan contento como en medio de un círculo; y esa alegría inalterable que se aprecia en él, no la obtiene de los que se le acercan, sino que la comunica».

${ }_{55}$ Los poemas de Voltaire «Sobre la Ley natural» y «Sobre el desastre de Lisboa», se incluyen en Alicia Villar, Voltaire-Rousseau: en torno al mal y la desdicha (Madrid: Alianza Editorial, 1995), 113-179.

${ }_{56}$ Cardano y Spinoza hablaban de los errores de la infancia y los prejuicios de la educación, y sus nombres aparecían en el «Poema sobre la Ley Natural» de Voltaire. Cfr. Villar, Voltaire-Rousseau: en torno al mal y la desdicha, 122.

57 J. J. Rousseau, Cartas a Sofía, 123.

58 J. J. Rousseau, Cartas a Sofía, 124

59 J. J. Rousseau, Cartas a Sofía, 129. Antes de escribir «innato», Rousseau ha dudado entre «eterno» y después «inmortal».
} 
para alcanzar la virtud, pero ahora la fuente de la felicidad se traslada a la interioridad del propio sujeto que descubre por introspección lo que es bueno o malo.

\section{LA EDUCACIÓN MORAL Y LAS MÁXIMAS DE LA PIEDAD}

Mientras que en el segundo Discurso se presentaban las alteraciones y pérdidas sufridas por las colectividades humanas, el Emilio ${ }^{60}$ plantea las adquisiciones de un individuo, un varón que crece al resguardo de una sociedad corrupta y que logrará abrirse camino en el mundo. Y en esta serie de adquisiciones aparece de nuevo la compasión. Me parece obligado aludir al papel secundario que Rousseau asigna a la mujer, encarnada en Sofía ${ }^{61}$ y del que han dado buena cuenta los estudios de género, una contradicción que no puede pasarse por alto en el defensor de la libertad, la igualdad y la autonomía. De este modo, se comprueba en este punto la coexistencia de esquemas innovadores con esquemas tradicionales que en tantos momentos se observa en las obras de Rousseau.

El método que sigue en el Emilio para conocer al ser humano es distinto al seguido en el segundo Discurso. En el libro IV dice fundarse en la observación, en lo que ha visto que es común a todos los hombres, en cualquier edad, rango o nación.

Frente a la educación tradicional, Rousseau se distancia de aquéllos que sólo veían a los niños como adultos en miniatura, y reivindica la infancia en sus propios modos de ver, pensar y sentir. «Dejad madurar la infancia en los niños», será uno de los legados de su obra, convencido de que la educación debe respetar y fortalecer el desarrollo natural y progresivo de las distintas facultades: sentidos, imaginación y razón. Si el ser humano es originariamente bueno y amante de la justicia, la «educación negativa», desde el punto de vista del educador o preceptor, consistirá en proteger al corazón del vicio y al espíritu del error. Se concretará en ejercicios más

\footnotetext{
${ }^{60} \mathrm{El}$ preceptor será un ser excepcional que acompañará a su alumno durante veinticinco años en su descubrimiento progresivo del mundo y le enseñará a guiarse por tres fuentes de educación: la naturaleza, las cosas y los seres humanos, para que aprenda de un modo gradual y a través de la propia experiencia, guiando a Emilio en su expansión y escogiendo sus relaciones o distintos escenarios.

${ }^{61}$ Precisam es el nombre que Rousseau da a la mujer que describe en el último libro del Emilio como bien nacida, de una bondad natural y de corazón sensible. Rousseau cae en el tópico de que «la mujer está hecha para gustar al hombre».
} 
que en dar preceptos, orientará progresivamente al educando hacia la autonomía más que hacia el servilismo; fortalecerá más que ablandará, pues para cerrar las puertas al vicio, es preciso combatir su puerta de entrada: la debilidad. El mejor educado será el que sepa soportar con fortaleza los males y los bienes que le depara la vida, y encuentre el modo de armonizar las distintas facetas de su ser: física, intelectual, afectiva y moral. Para Rousseau la etapa de la adolescencia, edad de las pasiones y la razón, implica un nuevo nacimiento que requiere una orientación moral. Más que adoctrinar, el objetivo de esta educación será orientar las experiencias del educando, con el fin de orientar las energías del joven hacia los sentimientos humanitarios.

La piedad que también se admitía en el animal en el segundo Discurso no se reconoce en los primeros años del niño. Curiosamente, aunque es uno de los primeros movimientos de la naturaleza, permanece dormida en tanto la imaginación no despierte. En el Discurso sobre el origen de las lenguas también defiende la importancia de la imaginación y la reflexión para poder experimentar la compasión. Considera que quien nunca ha reflexionado nunca podrá ser ni clemente, ni justo, ni piadoso, pues las afecciones sociales sólo se desarrollan con nuestras luces:

¿Cómo nos dejamos conmover por la piedad? Transportándonos fuera de nosotros mismos, identificándonos con el ser sufriente. Sólo sufrimos cuando juzgamos que él sufre; no es en nosotros es en él en quien sufrimos. ¡Piénsese cuánto supone de conocimientos humanos esta traslación! ¿Cómo imaginaría males de los que no tengo ninguna idea? ¿Cómo sufriría viendo sufrir a otro si no sé siquiera que sufre, si ignoro lo que hay de común entre él y yo? [...].Quien nada imagina nada siente más que a sí mismo: está solo en medio del género humano. ${ }^{62}$

Del mismo modo, en el Emilio también observa que para que surja la piedad es preciso que el niño reflexione sobre los otros seres sensibles que ha visto sufrir y morir, dice ahora Rousseau. De ahí que no aborde la piedad hasta la etapa de la adolescencia, la edad de la conmiseración y de la generosidad. Precisamente, la compasión hacia los que sufren es el pri-

${ }^{62}$ Jean -Jacques Rousseau, Discurso sobre el origen de las lenguas (Madrid: Akal, 1980), 60. Traducción de Mauro Armiño. 
mer sentimiento relativo que «toca el corazón humano» según el orden de la naturaleza; pero es sólo un principio, una disposición o tendencia, que por tanto debe ejercitarse para fortalecerse. De lo contrario, el amor de sí, pasión natural por excelencia que debería satisfacerse con pocas necesidades, deriva quizá en lo que hoy llamaríamos un egocentrismo narcisista. En cambio, cuando nos conmovemos por la piedad «nos transportamos fuera de nosotros mismos" y nos identificamos con el ser que sufre. De este modo, tomamos su ser y abandonamos el nuestro, nos descentramos. Es «en el otro en quien sufrimos», precisa en un texto del Emilio que Schopenhauer destacará, «no sufrimos más que en tanto juzgamos que sufre». ${ }^{63}$

Con el fin de nutrir esa sensibilidad naciente, el preceptor ofrecerá al joven experiencias que dilaten la fuerza expansiva de su corazón y la extiendan sobre otros seres. Pondrá a su alcance situaciones en las que ejercite su bondad, humanidad, compasión y beneficencia. Así, se impedirá que nazcan la envidia, la codicia, el odio y las pasiones crueles que dividen a los seres humanos y los hacen insensibles al dolor ajeno. Nada de vanidad, de emulación, de compararnos con los demás, son pasiones peligrosas que pueden llevar a odiar a quienes son más valorados que uno mismo.

Rousseau propone tres máximas, que comenta y califica como precisas, claras y fáciles de comprender. Y lo son, pero también ambivalentes, sobre todo la primera, pues su enunciado implica admitir una tendencia a la envidia: «No es propio del corazón humano ponerse en el lugar de personas que son más felices que nosotros, sino sólo de aquellas que son más de compadecer». ${ }^{64}$ En aplicación de su segunda máxima: «Nunca se compadecen en los demás sino los males de los que no nos creemos exentos», el preceptor tratará de avivar la imaginación del joven, para evitar una insensibilidad que le haría incapaz de ponerse en el lugar de otro. Deberá hacer comprender e imaginar a su alumno que la desgracia puede irrumpir en cualquier

\footnotetext{
63 «En efecto, ¿cómo nos podemos mover a la compasión si no es desplazándonos fuera de nosotros e identificándonos con el animal que sufre; dejando, por así decirlo, nuestro ser para tomar el suyo? No sufrimos sino en la medida en que juzgamos que él sufre: no es en nosotros, es en él donde sufrimos [...] debemos presentar al joven objetos sobre los que pueda obrar la fuerza expansiva de su corazón, que lo dilaten, que lo extiendan sobre los demás seres, que siempre le hagan reencontrarse fuera de sí; evitar con cuidado los que lo estrechan, lo concentran y tensan el resorte del yo humano» (Emilio o de la Educación, 298). Cf. Arthur Schopenhauer, «Sobre el fundamento de la moral», en Los dos problemas fundamentales de la ética (Madrid: Siglo XXI, 2002), 272, nota 110.
}

${ }^{64}$ Rousseau, Emilio o de la Educación. Véase el desarrollo de las máximas citadas en 298-301. 
momento, y que muchos acontecimientos imprevistos pueden cambiar el transcurso de una vida. La tercera máxima: «La piedad que tenemos del mal de otros no se mide por la cantidad de ese mal, sino por el sentimiento que atribuimos a quienes lo sufren», alerta sobre el peligro del endurecimiento. La memoria que nos hace sentir la continuidad de los males en el recuerdo, y la imaginación que nos lleva a anticipar el futuro, harán sopesar los efectos del sufrimiento y avivar la compasión.

Rousseau advierte que no trata de hacer al joven un hermano de la caridad, pero sí de evitar su impasibilidad ante las desgracias humanas, principio de la degradación moral. ¿Cómo y por qué medio? Primero, tratando de «sensibilizarle» con respecto a los que le rodean y alentando las relaciones de amistad y compañerismo que adquieren ahora un papel relevante para hacer germinar las primeras semillas de humanidad. También el conocimiento de la Historia, y sobre todo el contacto directo del joven con los que sufren desgracias, despertarán su sensibilidad moral. Nada es capaz de suplir la fuerza del testimonio directo, señala el ginebrino. Con ello, se promueve el ejercicio de la conmiseración, la bondad y la beneficencia: virtudes sociales por excelencia.

\section{DE LA BONDAD A LA VIRTUD}

Finalmente, Rousseau advierte que la piedad en su exceso puede degenerar bien en dureza, bien en debilidad, algo que no había observado en el segundo Discurso. Para impedirlo es preciso extenderla y generalizarla al género humano, observará ahora. Y aquí aparece de nuevo el tema de la justicia, señalando que es preferible entregarse a la piedad cuando está de acuerdo con la justicia. ${ }^{65}$ Así, estima que, de todas las virtudes, la justicia es la que más concurre al bien de los hombres, comprobándose así que resulta el concepto central de Rousseau, el fin supremo y la virtud decisiva. Como tal será invocada en sus escritos políticos junto con la virtud cívica, a la que apelaba desde su primer Discurso. ${ }^{66}$

Con ello, Rousseau busca el «justo medio» de la compasión, pues la piedad degenera cuando se limita a un mero contagio afectivo que nos de-

\footnotetext{
${ }^{65}$ Rousseau, Emilio o de la Educación, 339.

${ }^{66}$ Para un desarrollo del tema de la virtud cívica en Rousseau, cfr. Alicia Villar, «Democracia y virtud cívica en Montesquieu y Rousseau», en De la indignación a la regeneración democrática, coord. Olga Belmonte (Madrid: Universidad Pontificia Comillas, 2014), 71-90.
} 
bilita ${ }^{67}$ y extiende la tristeza por el mundo; se orienta debidamente cuando impulsa la acción y se relaciona con la justicia. ${ }^{68} \mathrm{La}$ bondad designa la propensión, la orientación fundamental de la voluntad hacia el bien, el orden y la justicia, y es una predisposición natural y favorable al ejercicio de la virtud, que exige decisión, esfuerzo, combate y la fuerza del alma. Como para Aristóteles, será la práctica, el hábito, lo que permitirá lograr una conducta virtuosa; de ahí la importancia de la educación, en un sentido integral. Por ello, el preceptor de Rousseau protegerá la bondad natural de su alumno, cerrando las puertas a la depravación, con el fin de que el niño se convierta en un adulto fuerte y autónomo, y se guíe por la virtud. Así, porque fue bueno, ante el conflicto de intereses, podrá ser virtuoso. La bondad es una tendencia, y dado que el ser humano es constitutivamente libre, no está determinado y puede tanto degenerar como perfeccionarse desde el punto de vista moral. Como se ha señalado, en sus obras políticas Rousseau hizo una llamada a la virtud cívica y a los sentimientos de ciudadanía más que a la bondad, pues la complejidad del estado social requiere la fuerza que caracteriza a la virtud. En sus últimas obras, Rousseau apela a la virtud que entiende como el vigor del alma; pues el hombre que sigue sus disposiciones o inclinaciones es naturalmente bueno, pero escuchando a su instinto y a su corazón, y no a su razón, no conoce la virtud que es trabajo y combate (Rousseau, juez. de Jean Jacques).$^{69}$ La virtud permitirá reinar sobre el propio corazón, y hacer sólo lo que es justo y honesto. ${ }^{70}$ En definitiva, para Rousseau es la libertad y la fuerza lo que hacen a los hombres excelentes, mientras que la debilidad y la esclavitud los convierte en malvados (Ensoñaciones del paseante solitario, $6 .^{\circ}$ Paseo $) .{ }^{71}$

\footnotetext{
${ }^{67}$ En el Emilio había advertido que toda maldad procede de la debilidad, de modo que considera que el niño es malvado porque es débil, y si se le convierte en fuerte, será bueno (Primer libro).

${ }^{68}$ Aristóteles define la compasión como «cierto pesar por la aparición de un mal destructivo y penoso en quien no lo merece, que también cabría esperar que lo padeciera uno mismo o alguno de nuestros allegados», Retórica, 1385b 15. Si en el segundo Discurso, la compasión surge inmediatamente ante la visión del sufrimiento ajeno, finalmente Rousseau vuelve a la definición canónica de Aristóteles que vincula la compasión con la observación de un sufrimiento inmerecido.

${ }^{69}$ Jean-Jacques Rousseau, Rousseau, juge de Jean-Jacques, en Oeuvres (Paris: Editions du Seuil, 1967), vol. I, 449.

${ }^{70}$ Rousseau, Rousseau, juge de Jean-Jacques, 448

${ }^{71}$ Jean- Jacques Rousseau, Ensoñaciones de un paseante solitario (Madrid: Cátedra, 1986), 111. Edición de F. J. Hernández
} 


\section{REFLEXIONES FINALES: LA AMBIVALENCIA DE LA COMPASIÓN}

A lo largo de la modernidad, la compasión tuvo valoraciones muy distintas en tanto fundamento de la moral. Por una parte, los racionalistas Descartes y Spinoza, advirtieron la ambivalencia de este sentimiento que extiende la tristeza y nos debilita, y prefirieron fundamentar la moral en los juicios firmes de la razón. ${ }^{72}$ Spinoza coincidió con Descartes en preferir impulsar los afectos alegres o positivos, en concreto la buena voluntad o la generosidad, que favorecen la benevolencia y la beneficencia.

Por otro lado, los moralistas británicos se distanciaron de la visión pesimista del ser humano que atendía fundamentalmente a su egoísmo y destacaron la importancia de los sentimientos naturales y de la simpatía que permiten ponerse en el lugar del otro. ${ }^{73}$ Cabe destacar en esta línea los nombres de Shaftesbury, Hutcheson, y posteriormente Adam Smith y David Hume. Algunas de las ideas de estos autores, muestran la proximidad con las defendidas por Rousseau. Sin poder detenerme en todos ellos, destacaré las de Shaftesbury, que en su Investigación sobre la virtud o el mérito ${ }^{74}$ traducida al francés por Diderot, distinguía dos clases de afecciones naturales: unas que inducen al bien público y otras que orientan hacia el interés particular. A su juicio, la propia naturaleza dicta estas afecciones para asegurar no sólo la conservación del individuo, sino también de la especie. Para Shaftesbury, un individuo no está desnaturalizado aunque pueda parecerlo, en tanto muestre una mínima inclinación hacia la amabilidad, la gratitud, la generosidad y la compasión. La ausencia total de esas afecciones, como se manifiesta en los que experimentan alegría o placer ante el sufrimiento ajeno, son propias de tiranos o de pueblos bárbaros. Shaftesbury se explica esa depravación en los individuos o en los pueblos, debido a la fuerza de las costumbres, pues la tendencia o disposición natural a la benevolencia puede ser corrompida por la fuerza de costumbres

\footnotetext{
${ }^{72}$ En general, los filósofos del siglo XVII desconfiaron de la piedad para fundamentar la moral, pues es un sentimiento de tristeza y temor, dirá Descartes, siguiendo la definición de Aristóteles, que disminuye nuestra capacidad de ser y pensar, añadirá Spinoza, inútil para quien vive bajo la guía de la razón (Etica, IV, prop. 50). Cfr. Alicia Villar, «El control racional de las pasiones: la ambivalencia de la compasión». Pensamiento, 64 (239), (2008): 143-150; Félix González Romero, Pasiones, amor y compasión en la construcción del sujeto moderno (Madrid: Universidad Pontificia Comillas, 2012).

${ }^{73}$ Sobre este tema, cf. Julio Seoane, Del sentido moral a la moral sentimental. El origen sentimental de la identidad y ciudadanía democrática (Madrid: Siglo XXI, 2014).

${ }^{74}$ Conde de Shaftesbury [Anthony Ashley Cooper], Investigación sobre la virtud o el mérito (Madrid: CSIC, 1997), 12-14. Estudio introductorio, traducción y notas de Agustín Andreu.
} 
contrarias. De ahí que insista, como después Rousseau, en la importancia de una buena educación para fortalecer y perfeccionar el sentido moral que puede corromperse. Como los moralistas británicos, Rousseau fue sensible a los aspectos positivos de la compasión y sólo ocasionalmente advirtió de sus peligros. En su caso, que degenere en debilidad o en dureza, o que resulte estéril y artificiosa, aspecto que desarrollará en su Carta a $D^{\prime}$ Alembert. ${ }^{75}$ "Nací sensible y bueno», confiesa Rousseau en sus Ensoñaciones de un paseante solitario, pero también se lamenta: «llevé la piedad hasta la debilidad». ${ }^{76}$

A lo largo del siglo XIX, Schopenhauer realizará una apología de la compasión que identificará con la caridad y el amor puro, mientras que Nietzsche, el crítico más feroz de este sentimiento que considera nada puro ni natural, desvelará sus lados más oscuros. Schopenhauer coincidirá con Rousseau en considerar que el rasgo esencial de la compasión es la identificación con el sufrimiento del otro. Permite la comprensión inmediata de que su desgracia podría ser la mía, y contrapesa el egoísmo natural, raíz de los enfrentamientos y rivalidades. Por este motivo, para ambos la compasión es el fundamento de la moral, pues al com-padecer suprimo, en cierto grado, la barrera o distancia que establezco con el otro y que me constituye en centro. Rousseau precisaba que la compasión "templa ${ }^{77}$ el egoísmo natural, y Schopenhauer advertirá que la ausencia de toda motivación egoísta o interesada es el criterio de la acción moral; de ahí que para ambos la compasión sea el origen de las acciones humanitarias y altruistas.

Muchos autores han alertado sobre la ambivalencia de la compasión, desde los estoicos a los racionalistas, pasando por Kant y Nietzsche, y más recientemente por Hannah Arendt. ${ }^{78}$ Kant, lector de Rousseau, coincidió con él en considerar a la compasión como un rasgo específicamente huma-

\footnotetext{
75 Esta ambivalencia se manifiesta también cuando Rousseau califica a la piedad como dulce por revelarnos la propia bondad, frente al sabor amargo de la envidia que da a conocer la propia miseria (Emilio, 296).

76 J. J. Rousseau, Ensoñaciones de un paseante solitario, 106.

77 J. J. Rousseau, Discurso sobre el origen y los fundamentos de la desigualdad entre los hombres, 235.

${ }^{78}$ En su obra Eichmann en Jerusalén. Un estudio sobre la banalidad del mal (Barcelona, Lumen, 2012) Hannah Arendt mostró con ejemplos de la historia que no hay que suponer que los malvados estén en conflicto consigo mismos. Observó que aunque el sadismo estuvo presente en los genocidios, quien de hecho organizó el asesinato masivo de seis millones de judíos fue una persona calificada como aparentemente normal por los psiquiatras, que se sentía mal si incumplía las órdenes recibidas.
} 
no que nos diferencia de los animales, ${ }^{79}$ pero discrepó en su función como fundamento de la moral. Al igual que la percepción en el conocimiento, la emoción en la moral debe ser cuidadosamente examinada y sólo será honesta cuando la razón reflexiva la haya elevado a deber. De ahí que las acciones benéficas deban realizarse por deber, no como respuesta a una inclinación compasiva. Para Kant, como para los racionalistas modernos, la compasión es expresión de debilidad, pasividad, inclinación y, a lo sumo es una obligación imperfecta.

Un siglo después, Nietzsche someterá a una crítica severa los sentimientos compasivos defendidos por el que había sido su maestro: Schopenhauer. Nietzsche desconfía de esa compasión empalagosa, ${ }^{80}$ que amarga nuestros goces terrestres y nuestra voluntad de vivir, que reproduce tristeza y debilidad, y denunciará las motivaciones «humanas, demasiado humanas» que pueden esconder los actos aparentemente altruistas. Mostró que es difícil que actuemos movidos por un solo motivo, sobre todo en el caso de la compasión, ${ }^{81}$ y que es imposible tratar de suprimir por completo el dolor de la vida, aspiración en ocasiones inconsciente del alma compasiva. También Hannah Arendt advirtió sobre la ambivalencia de la compasión y acudió a los testimonios que ofrece la Historia humana. En concreto, al caso de la Revolución francesa y la época del terror, donde «la piedad, considerada como resorte de la virtud; se demostró poseedora de un potencial de crueldad superior al de la propia crueldad». ${ }^{82}$

Las críticas mencionadas advierten de la complejidad de un sentimiento aparentemente simple, y señalan su ambivalencia, previniendo de los abusos e incluso de la manipulación ejercida en su nombre. ${ }^{83}$ Ahora bien, habría que preguntarse si esos riesgos anulan por completo su valor; de hecho,

\footnotetext{
${ }^{79}$ Immanuel Kant, Lecciones de Ética (Barcelona: Crítica, 1998), 269. Traducción de R. Rodríguez Aramayo y C. Roldán.

${ }^{80}$ F. Nietzsche, Más allá del bien y del mal, \& 33 (Madrid: Alianza Editorial, 1972), 58-59.

${ }^{81}$ F. Nietzsche, Aurora, \& 132 y \&133 (Madrid: Editores, S.L., 1994), 54-55. Sobre el significado de la piedad zarastustreana-nietzscheana, véase Remedios Avila, El desafío del nihilismo. La reflexión metafísica como piedad del pensar (Madrid: Trotta, 2005); sobre la afirmación de la vida por parte de Nietzsche, Diego Sánchez Meca, Nietzsche: la experiencia dionisíaca del mundo (Madrid:Tecnos, 4. ${ }^{a}$ ed., 2009).

82 Hannah Arendt, Essai sur la révolution (Paris: Gallimard, 1967), 127.

${ }^{83}$ Para un desarrollo de estos aspectos, cfr. Alicia Villar, «La ambivalencia de la compasión», en Pensar la compasión, ed. Miguel García-Baró (Madrid. Universidad Pontificia Comillas, 2008), 19-71.
} 
la compasión sigue estando presente en las éticas laicas, ${ }^{84}$ y en las llamadas a la solidaridad con las víctimas de catástrofes y atentados. Nos hace sentirnos concernidos ante el desamparo del otro, ante una experiencia de sinsentido o de absurdo que pide una respuesta, de ahí que pueda convertirse en el origen de las acciones humanitarias. Promueve el acercamiento y la cooperación, más que el enfrentamiento y la rivalidad, la conciencia del «nosotros» más que de la exclusión, e impulsa la reparación de una injusticia o de un acto de violencia que no puede quedar impune. Aún hoy, solemos calificar de inhumano al despiadado.

Sin duda, en nombre de la compasión, como en nombre de tantas palabras se pueden cometer engaños y abusos, pero es preciso contrarrestar una cierta práctica en la historia del pensamiento que privilegia un término en detrimento de otro. Como emoción no da una solución a los problemas, pero arraiga nuestras decisiones morales y contrarresta la insensibilidad y la indiferencia. Es evidente que en la vida y en la educación, sentimientos y razón no tienen que oponerse sino complementarse, pues lo opuesto a lo emocional no es tanto lo racional como la incapacidad de sentirse afectado. En el caso de la compasión, el discernimiento racional es preciso para advertir sus ambivalencias, sus sombras y excesos. ${ }^{85}$ Además, si bien la compasión y el amor a la justicia hacen sentirse concernido por la desgracia ajena, es preciso el análisis racional para evaluar los daños y las posibilidades reales de ayuda. El discernimiento racional nos alerta sobre las palabras o las acciones que pueden estar de más ante la desgracia ajena; nos previene de una compasión ciega que puede ser experimentada por el compadecido como una intromisión, o incluso como una humillación. Los sentimientos, reveladores tanto de la grandeza como de la miseria humana, pueden contribuir a articular los objetivos que definen la humanidad, como no dañar y promover el bien, pero la razón desempeña una función moderadora cuando obtener el fin se busca a cualquier precio y por cualquier medio.

\footnotetext{
${ }^{84}$ Presente en autores tan distintos como Levinas, Simone Weil, André Comte-Sponville, Richard Rorty, Martha Nussbaum o Valdimir Jankélévicth. Para una panorámica de los sentimientos y la compasión desde el punto de vista filosófico y ético, véanse, entre otros, el libro de Aurelio Arteta, ya citado, así como los de Joan-Carles Melich, Etica de la compasión (Barcelona: Herder, 2010), Victoria Camps, El gobierno de las emociones (Barcelona: Herder, 2011) y Jacinto Choza, Historia de los sentimientos (Sevilla: Thémata, 2011).

${ }^{85}$ Como los críticos de la compasión advirtieron, puede ser expresión de un egoísmo encubierto o incluso, según Nietzsche, de una voluntad de dominio.
} 
Para terminar, se comprueba que para Rousseau el ser humano no tiene precio sino valor. Fue más sensible que Kant a descubrir esta verdad a través de la experiencia de la compasión, y no sólo exclusivamente por medio de la virtud y del deber, en los que también insistió. Intuyó la importancia de lo que hoy llaman «hábitos del corazón», ${ }^{86}$ y defendió la importancia de los sentimientos, también los sentimientos de ciudadanía, en el siglo de la razón. Odiaba la servidumbre como la fuente de todos los males del género humano, y denunció las condiciones políticas que encarnaban la dependencia, la desigualdad y la opresión. A pesar de sus contradicciones, ${ }^{87}$ el interés de las obras de Rousseau está en los temas que aborda. Sin renunciar a determinados elementos del pensamiento antiguo, ${ }^{88}$ está en el origen de una transformación de la cultura moderna, que potenció la reflexión sobre la alteridad y la importancia de los sentimientos en la educación y en la construcción del yo, como ha destacado Charles Taylor. Sus obras subrayan la importancia de la vida moral y alientan la denuncia de la corrupción, convencido de que cabe alguna esperanza mientras subsista el sentimiento de justicia en el corazón humano. Siglos después Schopenhuaer, deudor de Rousseau, sentenciará que la compasión es un signo constitutivo de humanidad, pues si somos superiores a los animales es porque somos capaces de apiadarnos de ellos.

\footnotetext{
${ }^{86}$ Robert Bellah y otros, Hábitos del corazón (Madrid: Alianza Editorial, 1989).

${ }^{87}$ Rousseau negó la libertad de conciencia a las mujeres, e impuso a los ateos su catecismo del ciudadano. Cfr. María José Villaverde Rico, «Intolerancia, coacción y fanatismo en un apóstol de la tolerancia: Rousseau» en Forjadores de la Tolerancia, eds. M. ${ }^{a}$ José Villaverde Rico y John Christian Laursen (Madrid: Tecnos, 2011), 257, y Rosa Cobo, Fundamentos del patriarcado moderno: Rousseau (Madrid: Cátedra, 1995).

${ }^{88}$ Sobre este aspecto, véase Denise Leduc-Fayette, Jean-Jacques Rousseau et le mythe de l'antiquité (Paris: Vrin, 1974).
} 


\section{Nota sobre la autora:}

Alicia Villar Ezcurra es Catedrática de Filosofía en la Facultad de Ciencias Humanas y Sociales de la Universidad Pontificia Comillas (Madrid), participa actualmente en un Proyecto de Investigación financiado por el Plan Nacional de Investigación del Ministerio de Ciencia y Tecnología español dedicado a «Los fundamentos filosóficos de la idea de solidaridad: amor, amistad y generosidad» (FF 12012/37670), la fase cuarta de un proyecto que comenzó hace más de diez años.

Interesada por los pensadores que analizan la existencia y las pasiones humanas, gran parte de sus publicaciones se han dedicado al pensamiento de Pascal, Rousseau, Unamuno y el tema de la compasión. Entre los dedicados a Rousseau destacan sus dos libros publicados en Alianza Editorial (Voltaire-Rousseau: en torno al mal y la desdicha, y Cartas a Sofía). Otros trabajos dedicados a Rousseau son: la introducción a este autor publicada en Ediciones del Orto; los artículos «La fuerza de la piedad y los sentimientos de humanidad en Rousseau» (Pensamiento, 60, 228); "La compasión en Rousseau y Kant» (Revista Portuguesa de Filosofía, 61). Participó como ponente en los Congresos Internacionales sobre Rousseau celebrados en Murcia (2012), y en la Universidad de Bretaña Occidental (Cap. «Une correspondance morale, en el libro Rousseau en toutes lettres». Rennes: Ed. Eric Francalanza, PUR, 2014). 